\title{
INVESTIGACIONES
}

\section{Creencias de los adultos sobre la emoción de los niños: una propuesta de exploración en contextos de diversidad cultural ${ }^{1}$}

\author{
Adult's beliefs about children's emotions: \\ a proposal for exploration in contexts of cultural diversity
}

\author{
Enrique Riquelme ${ }^{a}$,Edgardo Miranda-Zapata ${ }^{b}$, Amy G. Halberstadt ${ }^{c}$ \\ ${ }^{a}$ Universidad Católica de Temuco, Chile. \\ eriquelme@uct.cl \\ ${ }^{b}$ Universidad de La Frontera, Chile. \\ ${ }^{c}$ North Carolina State University, EEUU.
}

\begin{abstract}
RESUMEN
Las creencias que los padres tienen sobre las emociones de los niños subyacen a los patrones de socialización emocional. La cultura mediatiza esta socialización; sin embargo, este proceso es habitualmente ignorado asumiendo un patrón común de ideales afectivos para todos los niños aun en contextos de alta migración y de alta presencia de culturas originarias. Así, el saber sentir ha sido históricamente mantenido al margen y su estudio desde una visión que reconoce las expectativas y creencias de las familias de grupos minoritarios es incipiente. Son pocos los estudios en Latinoamérica que dan cuenta de las variaciones culturales en las creencias de la emoción y menos los instrumentos que permitan hacerlo. Este trabajo recoge la necesidad de una evaluación de las creencias de los padres sobre la emoción de los niños en contextos de diversidad cultural y principalmente de grupos minoritarios de la IX región de La Araucanía en Chile. Participaron 307 adultos mapuches y no mapuches madres, padres y profesores de niños entre 4 y 10 años de edad. Se realizaron análisis de invarianza de medida, integrando las dimensiones originales del PBACE y nuevas dimensiones propuestas para el instrumento. Los resultados indican buenos índices de confiabilidad y un buen ajuste de las dimensiones analizadas, todas las dimensiones alcanzan al menos la invarianza escalar parcial, exceptuando una de ellas. El manuscrito finaliza analizando las limitaciones y proyecciones del estudio al campo de la educación y psicología en escenarios de diversidad cultural migratoria y con presencia de pueblos originarios.
\end{abstract}

Palabras clave: creencias, cultura, emoción, mapuche, cuestionario.

\begin{abstract}
The beliefs that parents have about children's emotions underlie emotional socialization patterns; culture mediates this socialization, however this effect is usually ignored assuming a common pattern of affective ideals for all children even in contexts of high migration and high presence of original cultures. Thus, knowing how to feel has been historically kept aside and its study, from a vision that recognizes the expectations and beliefs of families of minority groups is incipient. There are few studies in Latin America that account for cultural variations in the beliefs of emotion and less the instruments that allow it to do so. This work gathers the need of an evaluation of the beliefs of the parents on the emotion of the children in contexts of cultural diversity and mainly of minority groups of the IX region of La Araucanía, Chile. The sample included 307 mapuche and non-mapuche mothers,
\end{abstract}

Esta investigación contó con el financiamiento del programa FONDECYT 1195916, "Educación familiar y escolar: socialización emocional en contextos de diversidad social y cultural". 
fathers, and teachers of children between 4 and 10 years of age. Measurement Invariance analyses were performed integrating the original dimensions of the PBACE and the new dimensions proposed for the instrument. Results indicate good reliability indexes as well as a good adjustment of the analyzed dimensions; all but one dimension reached at least partial scalar invariance. Limitations are noted, and future study in the fields of education and psychology to culturally diverse settings are suggested.

Key words: beliefs, culture, emotion, Mapuche, questionnaire.

\section{INTRODUCCIÓN}

Los mecanismos de socialización emocional de los niños están fuertemente arraigados en las creencias que los adultos tienen sobre lo pertinente o deseable de una forma particular de expresión o regulación de la emoción (Baker, Fenning y Crnic, 2011). Estas creencias regulan los comportamientos de los niños en el plano emocional a través del modelamiento de los adultos (de su propio comportamiento emocional), reacciones hacia las emociones de los niños, conversaciones con estos sobre las emociones y el control de los nichos ambientales que ellos permiten a los niños, entre otros mecanismos de socialización (Cole y Tan, 2015; Dunsmore y Halberstadt, 1997; Eisenberg, Cumberland y Spinrad, 1998; Eisenberg, Spinrad y Cumberland, 1998).

Estos mecanismos de socialización de la emoción, y por lo tanto las creencias sobre lo pertinente o adecuado de una emoción, se encuentran integrados en la cultura y momento histórico en que los padres educan a sus hijos (Chentsova-Dutton y Tsai, 2010; Halberstadt y Lozada, 2011; Tamir et al., 2016). Las culturas mediatizan los estados afectivos ideales $\mathrm{y}$, por lo tanto, las formas deseables de un comportamiento o las reglas de despliegue emocional (Tamir et al., 2016). Existen, por lo tanto, diferencias culturales en estos aspectos de la socialización de la emoción (Parker et al., 2012; Tsai, Louie, Chen y Uchida, 2007). De esta forma, el supuesto de base que orienta el manuscrito es que las creencias que los padres tienen sobre la emoción de los niños mediatizan los procesos de socialización de la emoción, y que estas creencias están a su vez construidas en contextos histórico-culturales donde han sido funcionales (Chen, Fu y Zhao, 2015; Cole y Tan, 2015; Fernández, 2005; Riquelme, Quilaqueo, Quintriqueo y Loncón, 2016).

En efecto, la cultura entrega herramientas que orientan lo adecuado o inadecuado de una forma particular de regulación emocional de cada uno de sus miembros. Cuentos y narraciones son un buen ejemplo de una herramienta de educación emocional en un plano socio-cultural (Keel, 2016; Riquelme y Munita, 2017; Riquelme, Munita, Jara y Montero, 2013; Tsai et al., 2007; Wege et al., 2014). De esta forma, la familia y la escuela, padres y profesores actúan como agentes de socialización, a su vez mantienen creencias sobre la emoción de los niños que pueden estar alineadas con las características de la cultura en la que se han desarrollado. Lamentablemente, diferencias en las creencias sobre lo adecuado o funcional del comportamiento emocional de un niño puede traducirse en sobrediagnósticos o exclusión cuando perdemos de vista el marco cultural en el que estas creencias se han construido, situación frecuente para niños migrantes y de pueblos originarios (Riquelme, Lavoie, Quilaqueo y Quintriqueo, 2017).

Las creencias de los adultos acerca de las emociones de los niños también parecen ser importantes para su papel en el desarrollo de la resolución de los niños y para hacer frente a las emociones negativas o, alternativamente, en el desarrollo y mantenimiento 
de comportamientos clínicamente relevantes. Por ejemplo, algunas creencias aparecen asociadas con la resolución de problemas y la búsqueda de apoyo emocional apropiado en situaciones emocionalmente difíciles (Halberstadt, Thompson, Parker y Dunsmore, 2008b); pero otros tipos de creencias están asociados con la depresión, ansiedad, labilidad o comportamiento oposicionista-desafiante de los niños (Dunsmore, Booker, Ollendick y Greene, 2016; Rogers, Halberstadt, Castro, MacCormack y Garrett-Peters, 2016). Así, la identificación de estas creencias puede contribuir en la predicción e intervención en los procesos de regulación emocional y ansiedad de los niños (Mesquita, Marinetti y Delvaux, 2012; Veilleux, Salomaa, Shaver, Zielinski y Pollert, 2015).

Las creencias sobre la emoción que tienen los padres podrían predecir las estrategias de regulación de las emociones de los niños y subyacen a las prácticas de socialización de estos, prácticas que varían según el contexto social y cultural. Sobre este punto existe todavía un gran vacío teórico en el contexto sudamericano y vinculado a lo anterior, lamentablemente es posible también observar un vacío metodológico sobre el cómo (y el porqué) explorar las creencias sobre la emoción.

Chile ha sido un país diverso culturalmente, la presencia de pueblos originarios ha sido identificada históricamente, aunque no necesariamente reconocidos en sus saberes vernáculos; también desde el año 2000 la diversidad cultural se ha incrementado sostenidamente. En el 2017, según la encuesta de caracterización socioeconómica, el porcentaje de inmigrantes llegaría a 746 mil personas, el equivalente a un $4,35 \%$ del total de la población de Chile. Son principalmente personas provenientes de Perú, Colombia, Venezuela y Haití quienes contribuyen mayoritariamente a este porcentaje, pero fundamentalmente contribuyen con un saber sentir emocional y una serie de creencias sobre el ideal afectivo de los niños que subyacen a su quehacer cotidiano.

A pesar de los avances en nuestro entendimiento de las creencias sobre la emoción de los padres en contextos de diversidad cultural, los estudios sobre creencias y emoción se han llevado a cabo principalmente en Europa, Norteamérica y Asia (González, 2012; Parker et al., 2012; Raval y Martini, 2009; Raval, Raval, Salvina, Wilson y Writer, 2012). La riqueza de la diversidad cultural que caracteriza Sudamérica requiere con urgencia relevar este campo de investigación. En efecto, hasta ahora son muy pocos los estudios que exploran los vínculos entre cultura y emoción, y son aún más escasos aquellos estudios que exploran las creencias sobre la emoción de los niños desde el punto de vista de los adultos, en particular de los padres y profesores (para excepciones ver Jaramillo, 2013; Riquelme et al., 2016; 2017). Como hemos señalado, en conjunto con los dinámicos procesos migratorios, Chile se caracteriza por una alta presencia estable de pueblos originarios que todavía mantienen vivo su conocimiento y cultura, a pesar de lo anterior, la tendencia sigue siendo la del excluir un análisis de las emociones de los niños desde una perspectiva cultural y una evidente tendencia a la homogenización (Riquelme et al., 2016).

Dada la variedad de resultados relacionados con diferentes tipos de creencias, un paso necesario hacia la exploración de lo que se cree sobre la emoción requiere de una medida que permita explorar adecuadamente sus distintas variaciones y pueda ser aplicada de manera eficiente por profesionales e investigadores en psicología (por ejemplo, en las áreas clínica y del desarrollo), pero también en el campo de la educación (por ejemplo, padres, profesores, educadores diferenciales y orientadores, entre otros) y salud (embarazo y parto, programas de atención temprana, etc.). 


\section{CREENCIAS SOBRE LA EMOCIÓN DE LOS NIÑOS Y MODELOS DE EVALUACIÓN}

La socialización emocional de los padres hacia sus hijos se refiere a los esfuerzos de figuras parentales para guiar el desarrollo de las capacidades emocionales de los niños (Denham, Bassett y Wyatt, 2007). Estos esfuerzos incluyen el enseñar a los niños cómo etiquetar e interpretar las emociones, regular la expresión apropiada de la emoción y cómo regular la activación emocional (Cole y Tan, 2015). En efecto, se han evidenciado que estos esfuerzos de socialización están guiados por las creencias de los padres sobre la emoción de los niños (Dunsmore y Halberstadt, 2009; Halberstadt et al., 2008a), estas creencias sirven a los padres para guiar las conductas de sus hijos, y también hacia los niños en general, en tanto tienen un significado compartido en un plano social y cultural (McGillicuddy-DeLisi, 2009). Para Sigel y Kim (1996) las creencias de los padres son,

representaciones mentales que funcionan como una fuente de influencia sobre la conducta porque están conceptualizadas como los significados mediacionales que incluyen todas las facetas de la crianza, por ejemplo, los objetivos de los padres, enseñanza y manejo de las interacciones o estándares por los cuales se evalúa el desempeño de los niños (p. 85).

Probablemente existen muchas creencias distintas sobre las emociones (Dunsmore et al., 2009; Parker et al., 2012; Tamir et al., 2007), Por ejemplo, Gottman, Katz y Hooven (1997) enfatizaron dos tipos de filosofías de la emoción de los padres en la socialización emocional de los niños: aquellas relacionadas con el "entrenamiento" (coaching) emocional y las filosofías que evitan/rechazan (dismissing) la emoción. Los padres cuyas prácticas están más asociadas a la primera categoría, se definen por tener comodidad con la emoción y una visión positiva acerca de las emociones de sus hijos, en la creencia de que los acontecimientos emocionalmente cargados dan oportunidades para la intimidad y la enseñanza; para Ramsden y Hubbard (2002) específicamente, esta filosofía implica un foco en el que los padres logran darse cuenta de la emoción de sus hijos y la aceptación de sus emociones. Como efecto asociado de la práctica de esta filosofía, los niños tienen más probabilidades de ser emocionalmente regulados y socialmente competentes (Dunsmore, Booker y Ollendick, 2013; Rosso, 2012).

Los padres que utilizan la filosofía de "evitar/rechazar" la emoción tienden a descartar la idea de que las emociones tienen potencial como oportunidades de aprendizaje de los niños; en particular enfatizan la idea de que las emociones negativas son perjudiciales para el niño. Tales padres animan a los niños para deshacerse de la emoción sin exploración y creen que ello hará poco daño (Gottman et al., 1997). En la práctica de esta filosofía los niños tienden a la desregulación emocional y problemas de comportamiento (Dunsmore et al., 2016; Rosso, 2012; Rogers et al., 2016).

De Castella et al. (2013) plantean además una dimensión asociada a la controlabilidad o maleabilidad de las emociones, por ejemplo, señalan que algunas personas creen que estas no pueden ser cambiadas (teóricos entity), mientras que otros creen que las personas pueden aprender a controlar o regular las emociones (teóricos incrementales). Al igual que en el modelo de Gottman et al. (1997) la dimensión señalada en De Castella et al., (2013) también tiene importantes implicaciones para la autorregulación, para el funcionamiento 
social, así como para la socialización emocional. Quienes mantienen una creencia asociada a la estabilidad de las emociones, tienden a realizar juicios globales sobre las personas basadas en sus propias acciones; por ejemplo, si una persona en el plano emocional es considerada "débil", esa debilidad no podrá será superada. Tamir et al. (2007) en un estudio con estudiantes de pregrado, evidenció que aquellos que mantienen creencias "entity" sobre las emociones reportaban menores experiencias emocionales positivas y más experiencias negativas, así como mayores niveles de depresión, soledad y aislamiento.

Al contrario, quienes mantienen creencias incrementales de la emoción tenderán a dar más oportunidades de aprender a manejar las emociones a sus hijos y a sí mismos. De esta forma, aquellos que mantienen creencias incrementales sobre la emoción poseen mayor habilidad para regular sus propias emociones, en particular cuando son explícitamente instruidos para hacerlo (De Castella et al., 2013). En este sentido, las creencias sobre la emoción podrían ser un factor que explica diferencias individuales en el uso de estrategias de adaptación emocional como el reappraisal (Gutentag, Halperin, Porat, Bigman y Tamir, 2016).

Estos tipos de creencias de los padres dan cuenta del valor que implica explorar lo que los adultos creen sobre las emociones de los niños, creencias que subyacen al ideal afectivo y a la educación de la emoción que culturalmente damos a nuestros niños, pero también implica reconocer el valor de la forma en que las creencias se exploran. Así, la historia de las diferentes creencias está entrelazada con la historia de la medición de estas. De esta forma, revisaremos las investigaciones dedicadas a descubrir la riqueza de las creencias de los padres.

\subsection{MEDICIONES SOBRE LAS CREENCIAS SOBRE LA EMOCIÓN}

Las primeras formas de explorar las creencias sobre las emociones estuvieron centradas en relatos de los padres en la interacción con las emociones de sus hijos. En particular destacan los estudios de meta-emoción parental (Gottman et al., 1997) a través de entrevistas semiestructuradas que exploraban emociones, propias y de sus hijos, como la tristeza, el miedo y la ira, así como la forma de su hijo para hacer frente a las emociones.

Siguiendo con las contribuciones teóricas de este primer trabajo en meta-emociones, y reconociendo la necesidad de una medición con menor enfoque a la intensidad laboral, se desarrollaron varios cuestionarios. Dos derivan directamente de la teoría de la metaemoción y miden la aceptación o el rechazo de la emoción de los padres de una manera amplia (Hakim-Larson, Parker, Lee, Goodwin y Voelker, 2006; Lagacé-Séguin y Coplan, 2005). Otros, derivados de diferentes tradiciones teóricas, se centran en las creencias de los individuos sobre un aspecto amplio de la emoción o la regulación emocional, por ejemplo, si las emociones son peligrosas o pueden ser controladas e incluyen estudiantes universitarios, muestras de la comunidad o participantes con distintas etiologías; como el caso del síndrome de fatiga crónica (Dennis y Halberstadt, 2013; Rimes y Chalder, 2010; Vielleux et al., 2014). Estos cuestionarios presentan una tendencia a incluir agrupaciones de creencias más que a distinguir entre aquellas relacionadas y distintivas, centrándose en las creencias que tiene el adulto sobre sí mismo y sobre los demás.

A pesar de que los cuestionarios presentan fortalezas psicométricas prometedoras, preferimos cuestionarios que especifiquen creencias distintivas, ya que pueden entregar mayor validez predictiva y establecer estrategias de intervención con mayor dirección al 
momento de ser requeridas. También nos interesamos en particular por las creencias de los niños sobre las emociones. Ejemplos de precisión en la asesoría de la emoción incluyen creencias en el rol del lenguaje en la socialización de la emoción de los niños (Dunsmore y Karn, 2001) y la aceptación y consecuencias para los niños que demuestran una actividad negativa (Nelson, Leerkes, O'Brien, Calkins y Marcovitch, 2012).

Desarrollar una especificidad y multiplicidad de las creencias sobre las emociones de los niños es el objetivo del Parents Beliefs About Children's Emotion Questionnaire (PBACE) y sus variantes (Halberstadt et al., 2013). El PBACE es un cuestionario multidimensional que explora creencias organizadas en subescalas: a) el Costo de la positividad b) Valor de la rabia, c) Manipulación, d) Conocimiento parental, e) Control, f) Autonomía y g) Estabilidad.

Halberstadt et al., (2013) desarrollaron este instrumento como una revisión del Parents' Beliefs about Children's Negative Emotions Scale ([PBANE]; Halberstadt, Dunsmore, McElwain, Eaton, y McCool, 2001). Este instrumento incluía 80 ítems que abordaban distintas creencias de los padres sobre la expresión, experiencia, control y valor de las emociones de los niños; aunque el PBANE ha reportado buenos indicadores de validez (Wong, Diener, y Isabella, 2008; Wong, McElwain, y Halberstadt, 2009), no ha sido desarrollada directamente de los reportes de los padres respecto a las creencias, ni lo que es considerado como importante para ellos, tampoco ha sido probada su invarianza a través de género y la identificación cultural de los padres.

De este modo, los próximos pasos en el desarrollo del PBACE comenzaron con un análisis cualitativo previo de 12 grupos focales con muestras de los participantes representantes de 3 grupos culturales diferentes. Una vez que los ítems fueron generados de los grupos focales, el modelo fue probado mediante análisis exploratorios y factoriales confirmatorios, así como también la construcción de análisis de validez (Halberstadt et al., 2013). Las diferentes escalas del PBACE (listadas abajo) han demostrado indicadores positivos que encajan con el modelo, invariabilidad, consistencia interna, así como con la construcción de validez (Dunsmore et al., 2009; Halberstadt et al., 2008a; Halberstadt et al., 2013; Lozada, Halberstadt, Craig, Dennis, y Dunsmore, 2016; Stelter y Halberstadt, 2011; Uzbilir, 2016).

Con el objetivo de explorar las creencias de los padres sobre las emociones de los niños, y dentro de los contextos de alta migración y diversidad cultural, investigamos las propiedades psicométricas del PBACE en América Latina, particularmente en Chile; para luego continuar con expandir el PBACE e incluir las diversas creencias de los padres respecto de las emociones de los niños. Incluimos subescalas relevantes de creencias, según lo que pudimos identificar por los padres en una cultura única y minoritaria en Chile, la cultura mapuche. Al hacer esta adición también pudimos identificar creencias que podrían ser características de las familias mapuche en la IX región de la Araucanía. Por tanto, el objetivo de este estudio es explorar las propiedades psicométricas de la estructura multidimensional propuesta en el PBACE y proponer dimensiones que puedan ser integradas al instrumento para una exploración ajustada a las variaciones culturales en Chile. A este objetivo subyace la necesidad del reconocimiento del saber sentir de grupos minoritarios voluntarios o involuntarios (Ogbu y Simons, 1998), así los procesos migratorios y las culturas que forman parte de un nuevo escenario nacional deben ser reconocidas no solo en sus saberes escolares sino también en su saber sentir. 


\section{MÉTODO}

\subsection{CONTEXTO}

El estudio fue realizado en la IX región de La Araucanía en Chile. Esta región se caracteriza por ser un escenario de diversidad cultural que concentra un alto porcentaje de personas mapuche que corresponden al grupo minoritario involuntario (Ogbu y Simons, 1998) más numeroso en Chile. El último registro censal señala que, de los 16.634 .603 habitantes en Chile, el $9.1 \%$ pertenece a pueblos originarios, de los cuales el $84.4 \%$, se identifica como mapuche (Encuesta de Caracterización Socio-Económica, 2013). A pesar de los procesos de asimilación y aculturación asociados, entre otros, a la escolarización, las familias mapuche aún mantienen conocimientos vernáculos propios que forman parte de una educación que integra también formas deseables de ser y de sentir (Riquelme et al., 2016; 2017).

\subsubsection{Participantes}

Participaron 307 madres y padres mapuche y no mapuche de niños entre 2 y 14 años de edad de la IX región de La Araucanía, Chile. Las edades de los participantes oscilaron entre 18 y 63 años $(M=35,07 ; \mathrm{Dt}=9,98)$. Del total, 122 participantes $(39,7 \%)$, fueron mapuche y $185(60,3 \%)$ no mapuche. La muestra estuvo constituida por 51 hombres $(16,6 \%)$ y 256 mujeres $(83,4 \%)$. El proceso de selección de participantes fue por accesibilidad en comunidades mapuche de sectores rurales de la provincia de Cautín en la Región de la Araucanía. Todos participaron voluntariamente a través de consentimientos informados.

\subsubsection{Instrumento inicial}

Comenzamos con el Parents' Beliefs About Children Emotion ([PBACE], Dunsmore et al., 2009; Halberstadt, et al., 2013). Dicho instrumento explora creencias sobre las emociones de los niños desde el punto de vista de los padres. En el PBACE, las creencias sobre la emoción de los niños han sido organizadas en 7 subescalas que pueden ser utilizadas de manera independiente. Las subescalas y el por qué podrían ser relevantes para los procesos de socialización se describen a continuación:

a) El costo de la positividad de la emoción evalúa las creencias de los padres relacionadas con las potenciales consecuencias negativas de las emociones positivas de los niños. Se ha observado que los padres que creen que las emociones positivas de los niños pueden tener consecuencias negativas, habitualmente restringen el apoyo a las expresiones de alta activación emocional positiva de los niños (Halberstadt et al., 2013).

b) Valor de la rabia evalúa el grado en el cual los padres aceptan y valoran la rabia de los niños. Los padres que valoran la expresión de la rabia de sus niños también tienden a reconocerse como personas que expresan emociones negativas y, a su vez, apoyan este tipo de expresión emocional en sus hijos (Halberstadt et al., 2008a; Halberstadt et al., 2013; Lozada et al., 2016). Las dimensiones a y b se asocian a la evaluación de las emociones (Halberstadt et al., 2013).

c) Manipulación evalúa la creencia de que los niños usan las emociones para manipular 
a otros. Esta creencia de parte de los padres está asociada a la disminución de apoyo a la expresión de emociones de los niños (Halberstadt et al., 2013; Shipman y Zeman, 2001).

d) Conocimiento parental evalúa las creencias de los padres sobre la importancia de saber lo que le sucede en el plano emocional a los niños. Los padres que creen que deben conocer la vida afectiva de sus hijos apoyan más sus emociones negativas (Halberstadt et al., 2013). Las creencias de los padres sobre el rol de las emociones de los niños en las relaciones son evaluadas en las escalas c y d.

e) Control evalúa la creencia de que los niños son capaces de controlar sus emociones. La creencia de que los niños pueden controlar sus emociones se ha asociado con menos apoyo a la expresión de emociones negativas de los niños (De Castella et al., 2013; Halberstadt et al., 2013).

f) Autonomía evalúa la creencia de que los niños pueden aprender y manejar sus emociones sin la necesidad de apoyo de sus padres. Por ejemplo, padres que piensan que los niños pueden regularse a sí mismos sin apoyo parental, entregan menos apoyo a sus hijos cuando estos están tristes. Las creencias de los padres sobre las capacidades emocionales de los niños son exploradas a través de las dimensiones e y f.

g) Estabilidad explora las creencias de los padres sobre la estabilidad de las emociones de los niños a través de su desarrollo. Los padres que no ven que sus hijos cambian mucho con el tiempo son más negativamente expresivos y no respaldan las emociones de sus hijos (Halberstadt et al., 2013).

El instrumento PBACE original incluye 33 ítems, en los que se pregunta a los padres sobre su nivel de acuerdo en una escala Likert de 6 puntos organizados desde 1 (Completamente en desacuerdo) a 6 (Completamente de acuerdo).

\subsubsection{Procedimiento}

El proceso de adaptación del PBACE se llevó a cabo en tres fases:

1) Traducción y retrotraducción de ítems de la escala original: este proceso implicó la revisión de la traducción al español de hablantes nativos del inglés, así como la revisión de la traducción al inglés desde la versión en español. Esta fase buscó mantener la coherencia del contenido explorado en cada uno de los ítems de cada dimensión del instrumento de modo que las versiones fueran equivalentes en su contenido. Esta primera parte también implicó la revisión de los recientemente traducidos ítems al español por madres y padres con niños en etapa escolar con el fin de desarrollar un ajuste en el lenguaje y garantizar la comprensión y mantención del sentido original del ítem, de modo que la estructura profunda del instrumento pudiese ser equivalente con el original. Esta primera revisión se realizó con el objetivo de garantizar la adecuada comprensión de los reactivos y contribuir a la validez de contenido.

2) Integración de nuevas dimensiones: El PBACE ha evidenciado adecuados indicadores de validez en contextos occidentales, sin embargo, nada garantizaba que el instrumento incluyese aquellas creencias sobre la emoción relevantes para 
la familia chilena mapuche o no mapuche. En consideración a lo señalado, en un estudio previo se realizaron entrevistas semi-estructuradas, sobre los procesos de socialización emocional de los niños, a madres, a ancianos y también a niños mapuches en la Región de La Araucanía, Chile (Riquelme et al., 2017). En este estudio, a través de entrevistas semi-estructuradas, se exploraron, entre otras, dimensiones asociadas a los mecanismos de socialización de la emoción, ideales afectivos y cambios históricos en el plano de la educación emocional. Considerando que la cultura mapuche aún mantiene su lengua nativa mapuzungun, las entrevistas fueron desarrolladas en español con la asistencia de entrevistadores mapuche (mapuzungun/ español). Todas las entrevistas fueron luego transcritas para su posterior análisis. Los entrevistados recibieron una devolución de información para la validación del análisis realizado.

Riquelme et al. (2017) identificaron categorías que fueron recurrentes en los tres grupos, algunas de estas categorías sugirieron nuevas dimensiones del PBACE. Los ítems para estas nuevas dimensiones se redactaron usando las frases textuales de los mismos participantes, las que luego fueron revisadas por madres y padres (mapuche y no mapuche) con niños en etapa escolar, con el fin de garantizar la comprensión y mantención del sentido del ítem.

Las nuevas dimensiones emergentes en el estudio creencias chilenas fueron:

a) Control del miedo: esta dimensión explora creencias sobre la forma en que el miedo debe ser manejado por los niños. En particular la creencia de los padres es que esta emoción debe ser regulada en su expresión por los niños. Ejemplos de esta dimensión son: "el miedo es una emoción que los niños no deberían expresar". Las variaciones en las formas en que la emoción miedo es explicada y experimentada según la cultura han sido exploradas en investigaciones previas (Matsumoto, 1993; Muroff et al., 2014) que evidencian diferencias culturales en la forma de organizar esta emoción. Por ejemplo, en relación con las madres europeas, las madres áfrico-americanas señalaban que el despliegue de emociones como el miedo eran menos aceptables, además estas madres percibían mayores consecuencias sociales por el despliegue de estas emociones negativas (Nelson et al., 2012). En las entrevistas con los ancianos mapuche, la regulación de la emoción del miedo, así como su expresión, también emerge como uno de los temas centrales en la educación emocional de los niños. Así, en esta dimensión se han incluido ítems tales como: "El miedo es una expresión que los niños no deberían sentir", "Evitar sentir miedo hace al niño más seguro de sí mismo".

b) Valor del kumeche: Esta dimensión explora la idea de que las emociones de los niños deben ser auto-organizadas de modo que contribuyan a ser una persona íntegra, trabajadora, respetuosa, una "buena persona" que hace el bien y acciones a favor de su comunidad. Esta dimensión tiene un profundo anclaje en la idea del respeto como eje de la formación de personas (Dixon, Graber y Brooks-Gunn, 2008; García-Coll y Pachter, 2002). Sin embargo, el respeto como creencia sobre la emoción no se considera a menudo en la investigación clásica de la emoción que es el origen histórico del cuestionario de PBACE. 
El trabajo cualitativo previo (Riquelme et al. 2017), y de hecho nuestra propia experiencia anecdótica como chilenos, sugiere la centralidad de este concepto. En esta dimensión se han incluido ítems tales como: "Un niño sabio puede regular sus emociones" y "Los niños deben estar atento a las necesidades de los otros".

c) Importancia de los padres: Esta dimensión explora la creencia sobre la necesidad de que los niños usen, como referencia para regular sus emociones, a los padres y ancianos. Subyace a este principio la idea del respeto como eje articulador de la experiencia emocional en la interacción social con adultos y pares. El respeto en el plano afectivo a los padres y ancianos tiene además un profundo arraigo en la mantención de los conocimientos propios de la familia y cultura mapuche (Quilaqueo, Quintriqueo y Torres, 2016). En la educación familiar mapuche, la figura de los ancianos en la mantención y transmisión oral de los conocimientos ha sido clave; en este contexto la disposición activa de los niños a escuchar a los adultos (rumel allkutukey) es altamente valorada. La educación en el plano emocional se vincula entonces a esta necesidad de los niños a escuchar con respeto a los adultos y a los miembros de su comunidad. En esta dimensión se han incluido ítems tales como: "Los niños aprenden a regularse emocionalmente escuchando a los adultos" y "Una de las mejores formas de mostrar cariño a los padres es a través del respeto".

d) Conexión con la tierra: Esta dimensión explora la creencia sobre la utilización de la naturaleza como un regulador de la emoción de los niños. Es decir, la creencia de los padres de que los niños pueden interactuar con la naturaleza como un legítimo otro en la regulación de sus emociones. Como un componente más profundo de esta dimensión se destaca la creencia en los espíritus de la tierra (gnen) como los agentes que facilitan una forma particular de regulación de la emoción de los niños. Subyace entonces un componente espiritual central, propio del conocimiento familiar y cultural, asociado a la regulación de la emoción. En esta dimensión se han incluido ítems tales como: "La tierra [arboles, ríos, etc.] ayuda a los niños a regular su tristeza" y "La naturaleza puede ayudar a los niños a regular sus expresiones emocionales".

e) Tranquilidad: Esta dimensión explora la creencia que tienen los adultos sobre la capacidad del niño de mantenerse altamente regulado en el plano emocional, en control de sí mismo. En particular, la dimensión se vincula a la creencia sobre lo deseable de que los niños muestren disposición a escuchar en silencio y mantenerse tranquilos. Este ideal de comportamiento para los niños ha sido previamente descrito en Nicolaisen (1995) para la cultura Punan Bah de la isla Borneo, pero también la búsqueda de tranquilidad en los estados afectivos ideales ha sido descrita en las culturas asiáticas (Tsai, 2007). En esta dimensión se han incluido ítems tales como: "Un niño puede controlar mejor sus emociones cuando está tranquilo que cuando está hablando" y "Los niños deben expresar su alegría sin interrumpir a los demás".

3) Instrumento integrado: la nueva versión de PBACE incluye 12 dimensiones y 79 ítems, proporcionados en orden aleatorio. En la Tabla 1 es posible observar el número de ítems por dimensión. 
Tabla 1. Dimensiones e ítems

\begin{tabular}{|l|c|c|l|}
\hline Dimensión & $\mathrm{N}^{\mathrm{o}}$ & $\mathrm{N}^{\mathrm{o}}$ ítems & \multicolumn{1}{|c|}{ Ítems } \\
\hline Dimensiones originales & & & \\
\hline Autonomía & 1 & 7 & $2 ; 4 ; 7 ; 12 ; 14 ; 22 ; 28$ \\
\hline Conocimiento parental & 2 & 3 & $8 ; 20 ; 25$ \\
\hline Control & 3 & 5 & $11 ; 15 ; 21 ; 23 ; 70$ \\
\hline Costo Positividad & 4 & 4 & $26 ; 27 ; 31 ; 76$ \\
\hline Estabilidad & 5 & 4 & $5 ; 9 ; 16 ; 30$ \\
\hline Manipulación & 6 & 4 & $1 ; 17 ; 19 ; 32$ \\
\hline Valor de la rabia & 7 & 6 & $6 ; 10 ; 13 ; 18 ; 33 ; 73$ \\
\hline Nuevas Dimensiones & & & \\
\hline Control del Miedo & 8 & 12 & $3 ; 24 ; 29 ; 34 ; 39 ; 46 ; 49 ; 54 ; 67 ; 69 ; 71 ; 75$ \\
\hline Kumeche & 9 & 6 & $51 ; 55 ; 60 ; 63 ; 78 ; 79$ \\
\hline Padres & 10 & 7 & $38 ; 47 ; 52 ; 53 ; 58 ; 62 ; 64$ \\
\hline Tierra & 11 & 9 & $35 ; 37 ; 42 ; 43 ; 56 ; 57 ; 66 ; 68 ; 74$ \\
\hline Tranquilidad & 12 & 12 & $36 ; 40 ; 41 ; 44 ; 45 ; 48 ; 50 ; 59 ; 61 ; 65 ; 72 ; 77$ \\
\hline
\end{tabular}

\section{Análisis}

Análisis de invarianza de medida: Para los análisis factoriales confirmatorios, se consideró la escala de medida ordinal, de modo que se utilizó la matriz de correlaciones policóricas y el método de estimación de Robust Unweighted Least Squares (ULSMV). La matriz de correlaciones policóricas se recomienda si la totalidad de las variables es ordinal o algunas son ordinales y otras dicotómicas. La escala Likert del instrumento es ordinal, por lo tanto, se utilizó esta matriz de correlación (Finney y Distefano, 2006). Considerando las características señaladas, para evaluar el ajuste del modelo se emplearon: a) El estadístico Chi-cuadrado: teniendo en cuenta que el valor de $\chi 2$ es muy sensible a pequeñas desviaciones del modelo hipotetizado al trabajar con grandes muestras (Jöreskog, 1971; Jöreskog y Sörbom, 1993); b) El error cuadrático medio de aproximación (RMSEA); c) El índice Tucker-Lewis (TLI) y; d) El índice de bondad de ajuste comparativo (CFI) como medida de ajuste incremental. Hu y Bentler (1999) consideran valores aceptables del CFI, TLI superiores a .90, considerándose excelentes valores superiores a .95. Por último, un modelo fue considerado con un ajuste aceptable cuando el RMSEA presente valores menores a .07 (Steiger, 2007) y se valoró como un buen ajuste si el RMSEA es inferior a .05 (Batista-Foguet, Coenders y Alonso, 2004).

Para establecer el cumplimiento de la invarianza configural se utilizaron los criterios anteriormente señalados para los índices de bondad de ajuste. Para la satisfacción de la invarianza de medida, la invarianza escalar y la invarianza estricta, se consideró necesario que el Delta de Chi-cuadrado con corrección de Satorra-Bentler fuera no estadísticamente 
significativo (Satorra y Bentler, 2001). Se calculó la invarianza parcial cuando la invarianza escalar no fue alcanzada. En esos casos, se estimaron libremente los umbrales del ítem que presentó el mayor índice de modificación, superior a 3.84 (Dimitrov, 2010).

Se consideró que un ítem podía ser eliminado si presentaba un índice de modificación asociado por sobre 3.84. El análisis de confiabilidad se realizó por medio del coeficiente omega (McDonald, 1999), se consideró que un ítem podía ser eliminado si el valor de omega aumentaba al eliminar el ítem. Los análisis se realizaron usando los programas SPSS en su versión 20 (IMB CORP, 2011), Mplus en su versión 6.11 (Muthén y Muthén, 2007) y el paquete psych de la plataforma $\mathrm{R}$ versión 3.3.3.

\section{RESULTADOS}

Dadas las características del instrumento que permite el uso de cada subescala por separado (Halberstadt et al., 2013), en la Tabla 2 se muestran los resultados de la invarianza de medida y confiabilidad para cada subescala.

Dependiendo de los resultados de los distintos análisis realizados, se modificaron o descartaron diferentes ítems de las escalas. La Tabla 3 muestra la versión final del instrumento.

Se encontró que la subescala Autonomía, logró satisfacer una invarianza parcial escalar al liberar los umbrales del ítem 4. A pesar de ello, dado que la subescala se encontraba constituida por 3 ítems, se consideró poco adecuado sugerir la no consideración del ítem 4 para la comparación del constructo Autonomía entre niños mapuche y no mapuche, dado que la escala quedaría constituida solo por dos ítems. Así es que se valoró considerar el alcance de la invarianza métrica. Por lo que se puede utilizar la subescala en el grupo niños mapuche y no mapuche, lo cual permite comparar la relación de cada ítem con el constructo medido entre ambos grupos, pero no permite comparar el total de la subescala, ya que el origen de la escala puede diferir entre grupos (dado el incumplimiento de la invarianza escalar).

Las subescalas Costo Positividad, Manipulación y Kumeche, siendo escalas constituidas por 4 ítems, alcanzaron una invarianza parcial métrica al liberar los umbrales de uno de sus ítems. Se liberaron los umbrales del ítem 76 de la subescala Costo Positividad, los umbrales del ítem 1 de la subescala Manipulación y los umbrales del ítem 78 de la subescala Kumeche. Así, estas subescalas permiten comparar los valores para cada constructo medido para niños mapuche y no mapuche, sin considerar los ítems señalados para cada subescala.

La subescala Valor de la rabia, logra una invarianza escalar, por lo que permite que los valores para el constructo puedan ser comparados entre grupos de niños mapuche y no mapuche. Las subescalas Conocimiento parental, Control, Miedo, Padres, Tierra y Tranquilidad satisfacen la invarianza estricta, con lo que además de poder comparar los valores para cada constructo y estas subescalas permiten comparar los valores para cada ítem entre los grupos.

La subescala estabilidad no logra adecuados indicadores de ajuste en los grupos, específicamente no cumple los criterios para lograr la invarianza configural, de modo que la escala completa fue excluida de los análisis de confiabilidad posteriores.

Finalmente, se evidencia que todas las subescalas presentan buenos niveles de confiabilidad, con valores omega que oscilan entre 0.60 a 0.87 . 
Estudios Pedagógicos XLV, $\mathrm{N}^{\circ}$ 2: 61-81, 2019 CREENCIAS DE LOS ADULTOS SOBRE LA EMOCIÓN DE LOS NIÑOS: UNA PROPUESTA DE EXPLORACIÓN EN CONTEXTOS DE DIVERSIDAD CULTURAL

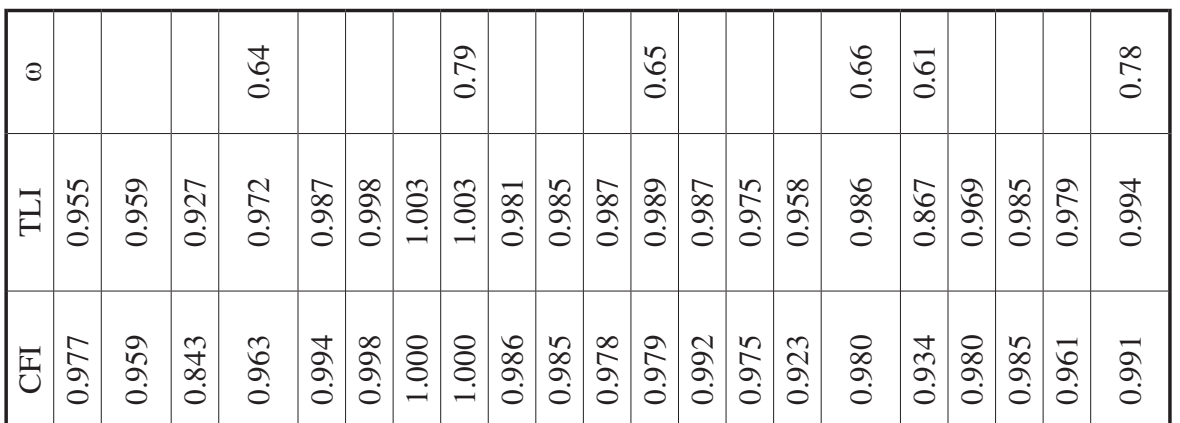

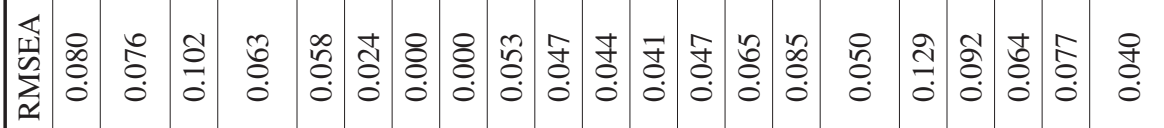

\begin{tabular}{|c|c|c|c|c|c|c|c|c|c|c|c|c|c|c|c|c|c|c|c|c|c|}
\hline & & & $\mid \begin{array}{c}\hat{8} \\
\tilde{v} \\
\tilde{b} \\
\dot{v} \\
\tilde{v}\end{array}$ & $\begin{array}{l}\hat{\jmath} \\
\stackrel{2}{g} \\
\stackrel{g}{+}\end{array}$ & & 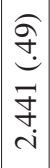 & $\mid \begin{array}{l}0 \\
0 \\
0 \\
0 \\
0 \\
\infty \\
0 \\
0\end{array}$ & $\mid \begin{array}{c}\hat{\sigma} \\
\dot{g} \\
\bar{a} \\
\hat{i}\end{array}$ & & 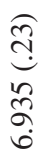 & 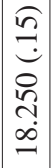 & $\begin{array}{c}\widehat{\vec{n}} \\
\infty \\
\tilde{n} \\
\tilde{n} \\
\end{array}$ & & 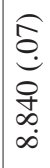 & 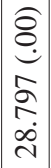 & 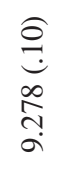 & & & 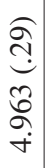 & 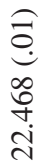 & 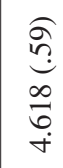 \\
\hline & & & 8 & $\simeq$ & $\vec{\imath}$ & $\hat{n}$ & $\ddot{n}$ & $\overline{0}$ & 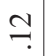 & \pm & $\simeq$ & \pm & ก̦ & so. & 8 & \pm & $\overline{0}$ & $\delta$ & o & $\overline{0}$ & $\bar{\imath}$ \\
\hline
\end{tabular}

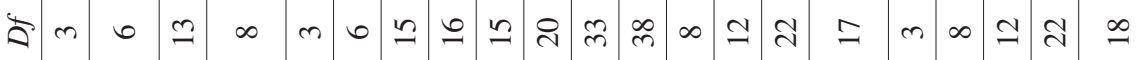

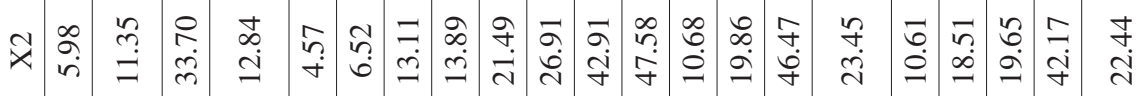

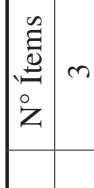

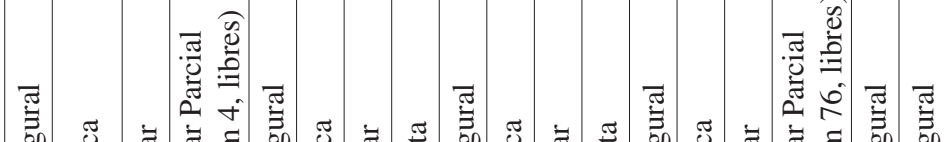
吾

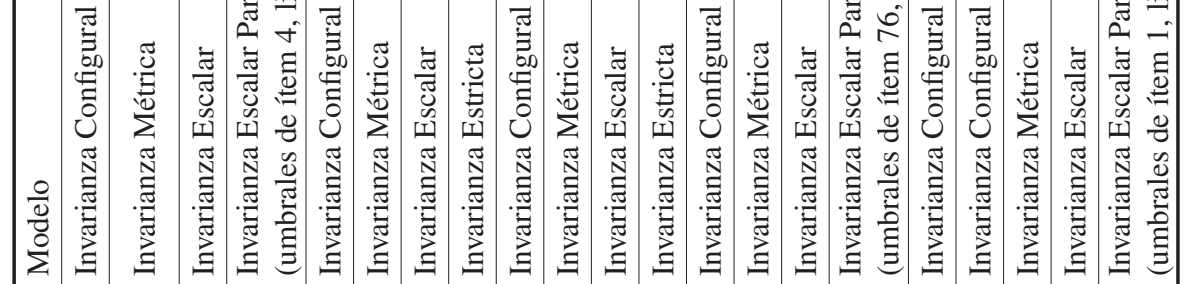

\begin{tabular}{|c|c|c|c|c|c|c|}
\hline 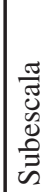 & 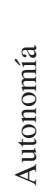 & 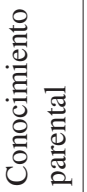 & $\begin{array}{l}\overline{0} \\
\overline{0} \\
\text { O0 }\end{array}$ & 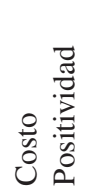 & 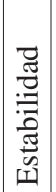 & 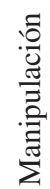 \\
\hline
\end{tabular}




\begin{tabular}{|c|c|c|c|c|c|c|c|c|c|c|c|c|c|c|c|c|c|c|c|c|c|c|c|}
\hline & & & $\stackrel{n}{n}$ & & & & $\stackrel{8}{\circ}$ & & & & 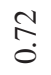 & & & & $\stackrel{m}{0}$ & & & & $\stackrel{\infty}{\infty}$ & & & & : \\
\hline ふু & ڤ్ & $\frac{\mathfrak{m}}{\hat{\sigma}}$ & $\begin{array}{l}\stackrel{g}{+} \\
\stackrel{0}{0}\end{array}$ & $\begin{array}{l}\stackrel{0}{ } \\
\stackrel{2}{0} \\
0\end{array}$ & $\begin{array}{l}\infty \\
\infty \\
0 \\
0\end{array}$ & $\begin{array}{l}t \\
\sigma \\
\sigma\end{array}$ & 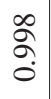 & $\begin{array}{l}\text { ț } \\
\stackrel{0}{0}\end{array}$ & $\begin{array}{l}2 \\
\approx \\
\vdots\end{array}$ & $\begin{array}{l}\tilde{1} \\
\infty \\
\vdots \\
0\end{array}$ & 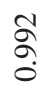 & 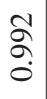 & $\frac{n}{\sigma}$ & $\vec{\sigma}$ & $\begin{array}{l}\infty \\
\stackrel{\circ}{\circ}\end{array}$ & $\begin{array}{l}\stackrel{2}{\sigma} \\
\text { }\end{array}$ & $\hat{\circ}$ & $\begin{array}{l}\check{\sigma} \\
\sigma\end{array}$ & $\begin{array}{l}\infty \\
\stackrel{\sigma}{\sigma} \\
\vdots\end{array}$ & $\begin{array}{l}\hat{\Omega} \\
\hat{\sigma}\end{array}$ & $\begin{array}{l}\bar{\sigma} \\
\grave{0}\end{array}$ & $\begin{array}{l}\stackrel{\circ}{\circ} \\
\text { }\end{array}$ & $\begin{array}{l}\text { مे } \\
\text { }\end{array}$ \\
\hline $\begin{array}{l}\text { ర్ } \\
\text { o. }\end{array}$ & ڤু & 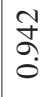 & $\begin{array}{l}\infty \\
\infty \\
\infty \\
0 \\
0\end{array}$ & $\begin{array}{l}n \\
\infty \\
0 \\
0\end{array}$ & $\begin{array}{l}\infty \\
\stackrel{\infty}{0} \\
\stackrel{0}{0}\end{array}$ & $\bar{\sigma}$ & ڤু & $\begin{array}{l}0 \\
\vdots \\
0 \\
0\end{array}$ & $\begin{array}{l}0 \\
\stackrel{0}{0} \\
\stackrel{0}{0}\end{array}$ & 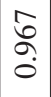 & 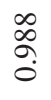 & $\begin{array}{l}\text { बे } \\
\text { }\end{array}$ & ڤু & $\begin{array}{l}\mathscr{2} \\
\stackrel{0}{0} \\
\stackrel{2}{0}\end{array}$ & $\begin{array}{l}\stackrel{2}{\partial} \\
\stackrel{0}{0}\end{array}$ & $\bar{\sigma}$ & $\hat{\grave{\alpha}}$ & $\stackrel{\check{\sigma}}{\circ}$ & $\begin{array}{l}\stackrel{2}{\sigma} \\
\stackrel{\sigma}{0}\end{array}$ & $\begin{array}{l}\bar{\infty} \\
\stackrel{0}{\circ}\end{array}$ & $\bar{\sigma}$ & 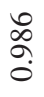 & ๙ু \\
\hline
\end{tabular}

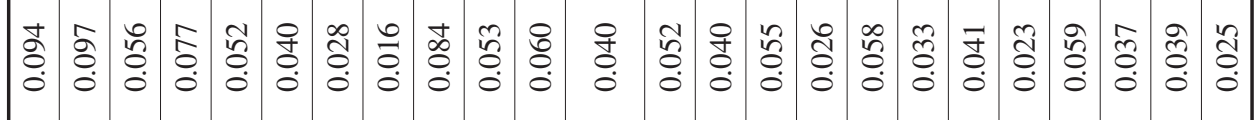

\begin{tabular}{|c|c|c|c|c|c|c|c|c|c|c|c|c|c|c|c|c|c|c|c|c|c|}
\hline & 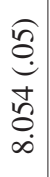 & 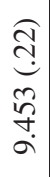 & $\begin{array}{l}\widehat{\sigma} \\
\dot{\sigma} \\
m \\
m \\
\dot{d}\end{array}$ & & 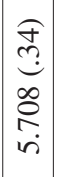 & 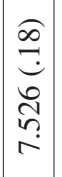 & $\begin{array}{l}\widehat{\tilde{n}} \\
\mathfrak{v} \\
\overrightarrow{0} \\
\infty \\
\dot{n}\end{array}$ & & $\begin{array}{l}\underset{n}{n} \\
\stackrel{\infty}{\infty} \\
\stackrel{\infty}{\approx} \\
\dot{m}\end{array}$ & 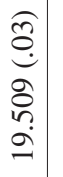 & 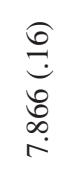 & & 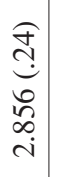 & 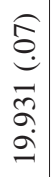 & 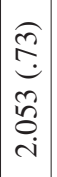 & & 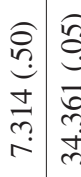 & 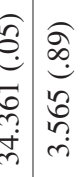 & & 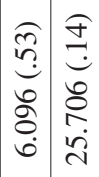 & 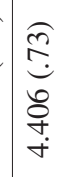 \\
\hline $\bar{o}$ & రิ & $\stackrel{\simeq}{\because}$ & $\overline{0}$ & $\stackrel{m}{\because}$ & $\vec{ヘ}$ & సุ & q. & ô. & \pm & $\stackrel{n}{\circ}$ & กิ & $\stackrel{\infty}{\longrightarrow}$ & $\stackrel{\Upsilon}{\Upsilon}$ & ô. & $\approx$ & $\overline{0}$ & $\stackrel{\infty}{\rightarrow}$ & 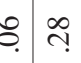 & ô. & $\because=$ & $\stackrel{\infty}{\sim}$ \\
\hline$m$ & 6 & $\cong$ & $\stackrel{\infty}{-}$ & $\cong$ & ก & $m$ & $\infty$ & $\infty$ & $\simeq$ & $\approx$ & I & $\infty$ & 으 & $\approx$ & i & $\stackrel{\infty}{+}$ & 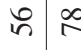 & $\infty$ & $\tilde{m}$ & I $\overline{6}$ & 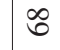 \\
\hline$\stackrel{g}{\stackrel{2}{r}}$ & $\begin{array}{l}\vec{b} \\
\dot{ \pm}\end{array}$ & $\begin{array}{l}\stackrel{1}{1} \\
\stackrel{a}{ }\end{array}$ & $\begin{array}{l}\vec{j} \\
\dot{m}\end{array}$ & 官 & $\begin{array}{l}8 \\
\infty \\
\dot{d}\end{array}$ & 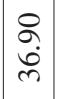 & $\begin{array}{l}\infty \\
\\
\stackrel{m}{n}\end{array}$ & 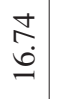 & $\begin{array}{l} \pm \\
\Xi\end{array}$ & $\begin{array}{l}8 \\
\dot{j} \\
\dot{m}\end{array}$ & $\stackrel{\stackrel{J}{\sim}}{\stackrel{\sim}{N}}$ & $\stackrel{n}{=}$ & $\vec{n}$ & $\begin{array}{l}\tilde{n} \\
\stackrel{2}{2} \\
\tilde{n}\end{array}$ & $\begin{array}{l}\stackrel{m}{\sim} \\
\infty \\
\dot{\sim}\end{array}$ & 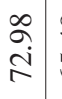 & $\begin{array}{ll}a & 8 \\
\dot{b} & \sigma\end{array}$ & 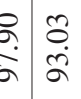 & $\begin{array}{l}\tilde{\sigma} \\
\tilde{n} \\
\end{array}$ & \begin{tabular}{l|l}
8 & $\infty$ \\
\hdashline & $\infty$ \\
$\dot{\vec{n}}$ & $\mathbb{+}$ \\
\end{tabular} & $\begin{array}{l}\text { P } \\
\text { ṫ }\end{array}$ \\
\hline$m$ & & & & in & & & & $\nabla$ & & & & $\nabla$ & & & & $\infty$ & & & $r$ & & \\
\hline 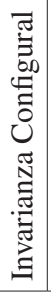 & 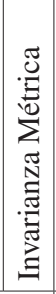 & 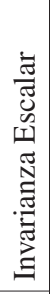 & 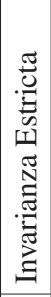 & 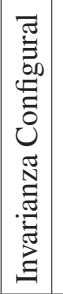 & 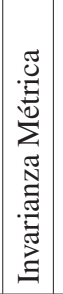 & 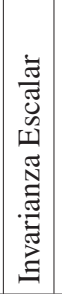 & 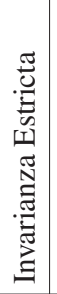 & 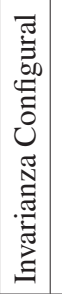 & 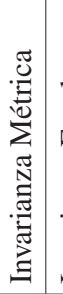 & 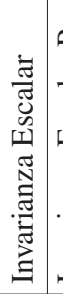 & 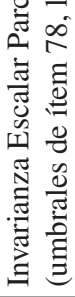 & 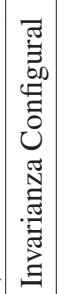 & 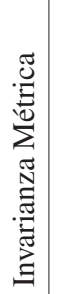 & 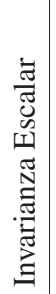 & 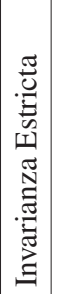 & 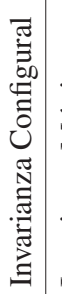 & 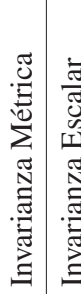 & 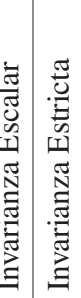 & 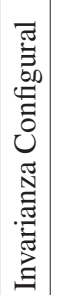 & 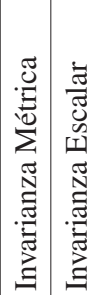 & 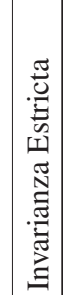 \\
\hline & t & & & & 월 & 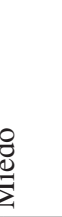 & & & 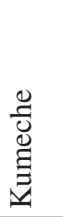 & & & & & & & & $\stackrel{\widetilde{E}}{\stackrel{D}{E}}$ & & & 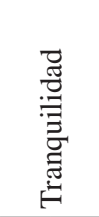 & \\
\hline
\end{tabular}


Tabla 3. Dimensiones e ítems del instrumento final

\begin{tabular}{|l|l|l|l|}
\hline Escala & $\mathrm{N}^{\mathrm{o}}$ & $\mathrm{N}^{\mathrm{o}}$ ítems & Ítems \\
\hline Dimensión original & & & \\
\hline Autonomía & 1 & 3 & $4 ; 12 ; 28$ \\
\hline Conocimiento parental & 2 & 3 & $8 ; 20 ; 25$ \\
\hline Control & 3 & 5 & $11 ; 15 ; 21 ; 23 ; 70$ \\
\hline Costo Positividad & 4 & 4 & $26 ; 27 ; 31 ; 76$ \\
\hline Manipulación & 5 & 4 & $1 ; 17 ; 19 ; 32$ \\
\hline Valor de la rabia & 6 & 3 & $6 ; 18 ; 73$ \\
\hline Nuevas dimensiones & & & \\
\hline Control del Miedo & 7 & 5 & $29 ; 34 ; 49 ; 67 ; 69 ; 71$ \\
\hline Kumeche & 8 & 4 & $51 ; 60 ; 78 ; 79$ \\
\hline Padres & 9 & 4 & $38 ; 52 ; 58 ; 62$ \\
\hline Tierra & 10 & 8 & $35 ; 37 ; 43 ; 46 ; 57 ; 66 ; 68 ; 74$ \\
\hline Tranquilidad & 11 & 7 & $40 ; 41 ; 45 ; 48 ; 50 ; 59 ; 72$ \\
\hline
\end{tabular}

\section{DISCUSIÓN Y CONCLUSIONES}

En el marco de un escenario de diversidad social y cultural, este estudio permitió entregar una herramienta que permite reconocer las creencias que subyacen a una forma de conocimiento sobre el saber sentir, que históricamente ha sido mantenido al margen de la educación formal bajo el supuesto de un ideal afectivo común para todos y todas los niños y niñas. En efecto, este estudio presenta una herramienta que permite romper la tendencia a homogeneizar e imponer conocimientos propios cuando la variación cultural se incrementa en contextos de migración y diversidad cultural. En particular el estudio valoró las propiedades del cuestionario PBACE (Halberstadt et al., 2013) para la exploración de las creencias de los adultos sobre las emociones de los niños. Además, el estudio propuso nuevas dimensiones que puedan ser integradas al instrumento para una exploración ajustada a las variaciones culturales en Chile.

En el proceso, desde un abordaje cualitativo, se integraron dimensiones que emergieron de análisis previos sobre relatos asociados a los procesos de socialización emocional, en los que subyacen creencias propias de las madres y sabios mapuche que viven en comunidades de la IX región de la Araucanía en Chile (Riquelme et al., 2017). Estas dimensiones, junto a aquellas propuestas en el modelo original de Halberstadt et al. (2013), fueron sometidas a análisis cuantitativos centrados en la invarianza de medida y análisis de confiabilidad.

Los resultados de este estudio resaltan nuevamente el valor de trabajar con padres y ancianos para identificar sus sistemas de creencias para que puedan crearse ítems que sean fieles a esas creencias. En nuestro caso intentamos utilizar su propio idioma con el objetivo 
de capturar de manera precisa sus creencias, y las probamos inicialmente devolviéndoselas a los padres mapuche y a los mayores mapuche y no mapuche.

Además, cabe señalar que las diferencias étnicas distintivas que emergieron en un grupo (mapuche) fueron conceptos comprendidos por los chilenos no mapuches, quienes entienden la misma estructura mental, a pesar de que las escalas puedan estar advocadas a diferentes grados (Halberstadt, Riquelme y Oertwig, s.f.). Se puede notar que los diferentes grupos aceptan la misma estructura logística (invarianza). Los resultados dan cuenta de un instrumento que permite realizar una adecuada exploración sobre las creencias de la emoción de los niños por parte de los adultos. Además, se aporta un instrumento que es sensible a las variaciones culturales presentes en Chile. De esta forma, se entrega un instrumento válido y confiable, que permite el uso de sus subescalas de forma independiente del resto de las subescalas del instrumento. Es necesario tener en cuenta que las escalas Valor de la rabia, Conocimiento parental, Padres, Control, Costo Positividad, Manipulación, Kumeche, Miedo, Tierra y Tranquilidad permiten la comparación de los valores en el constructo medido en niños mapuche y no mapuche. Por su parte, la subescala Autonomía debe limitarse a su uso en cada grupo de forma separada.

Los resultados de este estudio pueden ser analizados en, al menos, dos ámbitos: el primero de ellos se asocia al problema que subyace al estudio, es decir, a la necesidad del reconocimiento de las variaciones culturales en la regulación de la emoción. A pesar de que la evaluación de la variación cultural prevalece más allá del ámbito de este artículo, es una suposición implícita de nuestro trabajo. Este cuestionario aporta al creciente número de herramientas que han comenzado a evaluar estas premisas, favoreciendo una investigación más profunda del contexto cultural y su impacto en las creencias de los padres acerca de las emociones de los niños. Como tal, contribuye al creciente número de instrumentos que están disponibles para evaluar las influencias culturales y lo hace con dimensiones múltiples que son en gran parte invariantes a través de dos culturas principales en Chile, pero que permiten proyectar su aplicación en las distintas culturas que los escenarios de migración entregan.

En el presente estudio se integran escalas desarrolladas en contextos occidentales y encuentran apoyo para su uso en Chile, también pudimos identificar creencias centrales sobre la emoción que podrían ser de singular importancia para el contexto chileno (ya que no fueron descubiertos antes): el control del miedo, el respeto, la tierra como reguladora de las emociones, el rol de padres/ancianos y el ser kumeche son ejemplos de creencias propias sobre la emoción de los niños, vinculadas a ideales emocionales y modelos educativos que son relevantes tanto para mapuche como no mapuche.

El segundo punto es la necesidad de hacer un giro en la exploración de las creencias sobre la emoción en contextos de diversidad cultural, específicamente, desde el anticipar lo regular a esperar la variación. En efecto, una mirada puesta en la expectativa de cambio en los contextos culturalmente diversos ofrece mayores oportunidades para el reconocimiento de culturas y nuevos elementos que emergen como dimensiones propias en las creencias sobre la emoción. Así, las dimensiones originales del PBACE, así como las nuevas dimensiones que se han integrado, nos permiten ahora explorar si padres y profesores comparten o no la misma idea sobre las emociones de los niños. Esta exploración podría ser incorporada como potencial herramienta para el trabajo con los niños y con los adultos para explorar las variaciones.

Especialmente emocionante sobre estas nuevas escalas es la oportunidad ahora de explorar estos conceptos en muestras no mapuches e incluso no chilenas, evaluando su 
invarianza y el grado en que los padres y otros adultos respaldan estas creencias. Estas son creencias de importancia para los mapuches, pero también pueden ser de interés universal con resultados positivos para los niños cuando los padres respaldan estas creencias.

Todos queremos niños felices; sin embargo, las creencias sobre las tonalidades y variaciones culturales de esta felicidad pueden hacer la diferencia entre un niño ajustado a la cultura mayoritaria y un niño que es excluido por esta misma cultura. Por ejemplo, la dimensión tranquilidad en las creencias de la emoción de los niños puede ser un elemento clave en la expectativa de los adultos sobre la capacidad de regulación emocional de los niños. De la misma forma, la dimensión control, que explora cuánto es deseable para un adulto de una cultura que el niño logre tempranamente controlar sus emociones por sí mismo, tiene correlatos prácticos que pueden hacer la diferencia entre estimular para aumentar el control autónomo de las emociones de los niños en una cultura o, estimular para la regulación de las emociones de los niños desde los adultos en otra cultura. En particular la naturaleza, en su relación con la dimensión espiritual, emerge como un legítimo otro en la regulación de la emoción de los niños. Esta es una dimensión clave a considerar en la educación escolar. Así, cada una de las dimensiones de PBACE constituye una potencial fuente de exploración de las variaciones culturales de la emoción.

Considerando los puntos señalados, el presente manuscrito no solo entrega un instrumento para explorar creencias sobre la emoción, pretende relevar los elementos clave que subyacen al proceso de construcción de este: la cultura moldea la forma en que educamos las emociones de nuestros niños y acceder a las creencias sobre la emoción nos entrega una vía para entender aquellos elementos socioafectivos que son centrales en cómo entendemos nuestra historia y nos proyectarnos como sociedad.

Para finalizar, consideramos que el instrumento tiene proyecciones en distintos campos de acción; siguiendo a Halberstadt et al. (2013), diferentes creencias de los padres sobre las emociones de los niños podrían tener un impacto en distintas dimensiones del comportamiento de los niños. De esta forma, desde los campos clínicos, explorar estas creencias podría ser de utilidad en el diseño de estrategias de educación emocional para los padres desde el reconocimiento de marcos culturales. Así, focalizándonos en lo que los padres creen sobre la emoción podría ser más útil que la implementación de programas basados únicamente en la modificación de sus conductas.

Desde el campo de la educación escolar, es también fundamental la exploración del currículum emocional (Sala y Abarca, 2001) y, en particular, las creencias de los docentes que subyacen a sus prácticas de educación emocional en un currículum habitualmente oculto (Alisaari y Heikkola, 2017).

En contextos migratorios, conocer los ideales afectivos que subyacen a la educación emocional de los niños es clave. Este conocimiento, sin duda, disminuirá la discriminación y sobrediagnóstico, pero por sobre todo educará a los adultos sobre la riqueza de un saber sentir que varía culturalmente. El cuestionario que hemos presentado permite comenzar este trabajo.

\section{REFERENCIAS BIBLIOGRÁFICAS}

Alisaari, J. y Heikkola, L. (2017). Songs and poems in the language classroom: Teachers' beliefs and practices. Teaching and Teacher Education, 63, 231-242. 
Estudios Pedagógicos XLV, Nº 2: 61-81, 2019

CREENCIAS DE LOS ADULTOS SOBRE LA EMOCIÓN DE LOS NIÑOS: UNA PROPUESTA DE EXPLORACIÓN EN CONTEXTOS DE DIVERSIDAD CULTURAL

Baker, J., Fenning, R. y Crnic, K. (2011). Emotion socialization by mothers and fathers: Coherence among behaviors and associations with parent attitudes and children's social competence. Social Development, 20(2), 412-430. doi: 10.1111/j.1467-9507.2010.00585.x.

Batista-Foguet, J., Coenders, G. y Alonso, J. (2004). Análisis factorial confirrmatorio. Su utilidad en la validación de cuestionarios de salud. Medicina Clínica (Barcelona), 122(1), 21-27.

Castella de, K., Goldin, P., Jazaieri, H., Ziv, M., Dweck, C. S. y Gross, J. (2013). Beliefs about emotion: Links to emotion regulation, well-being, and psychological distress. Basic and Applied Social Psychology, 35(6), 497-505.

Chen, X., Fu, R. y Zhao, S. (2015). Culture and socialization. En J. Grusec \& P. Hastings (Eds.). Handbook of socialization: Theory and research ( $2^{\text {nd }}$ edition) (pp. 451-471). New York: Guilford Press.

Chentsova-Dutton, Y. y Tsai, J. (2010). Self-focused attention and emotional response: The role of culture. Journal of personality and social psychology, 98(3), 507-519.

Cole, P. y Tan, P. (2015). Emotion socialization from a cultural perspective. En J. Grusec y P. Hastings (Eds.), Handbook of socialization: Theory and research ( $2^{\text {nd }}$ edition) (pp. 499-519). New York: Guilford Press.

De Castella, K., Goldin, P., Jazaieri, H., Ziv, M., Dweck, C. S., y Gross, J. J. (2013). Beliefs about emotion: Links to emotion regulation, well-being, and psychological distress. Basic and applied social psychology, 35(6), 497-505.

Denham, S., Bassett, H. y Wyatt, T. (2007). The socialization of emotional competence. En J. Grusec y P. Hastings (Eds.), The handbook of socialization (pp. 614-637). New York: Guilford Press.

Dennis, P. y Halberstadt, A. (2013). Is believing seeing? The role of emotion-related beliefs in selective attention to affective cues. Cognition and Emotion, 27(1), 3-20.

Dimitrov, D. (2010). Testing for factorial invariance in the context of construct validation. Measurement and Evaluation in Counseling and Development, 43(2), 121-149. doi: $10.1177 / 0748175610373459$.

Dixon, S., Graber, J. y Brooks-Gunn, J. (2008). The roles of respect for parental authority and parenting practices in parent-child conflict among African American, Latino, and European American families. Journal of family psychology, 22(1), 1-10.

Dunsmore, J., Booker, J. y Ollendick, T. (2013). Parental emotion coaching and child emotion regulation as protective factors for children with Oppositional Defiant Disorder. Social Development, 22(3), 444-466. doi: 10.1111/j.1467-9507.2011.00652.x.

Dunsmore, J., Booker, J., Ollendick, T. y Greene, R. (2016). Emotion socialization in the context of risk and psychopathology: Maternal emotion coaching predicts better treatment outcomes for emotionally labile children with oppositional defiant disorder. Social Development, 25(1), 8-26. doi:10.1111/sode.12109.

Dunsmore, J. y Halberstadt A. (1997). How does family emotional expressiveness affect children's schemas? En: K. Barrett (Ed.), The communication of emotion: Current research from diverse perspectives (pp. 45-68). San Francisco: Jossey-Bass.

Dunsmore, J. y Halberstadt A. (2009). The dynamic context of children's emotions: Family and cultural system influences. En J. Mancini, K. Roberto (Eds.), Pathways of human development: Explorations of change (pp. 171-190). Boulder, Co: Lexington Books.

Dunsmore, J., Her, P., Halberstadt, A. y Perez-Rivera, M. (2009). Parents' beliefs about emotions and children's recognition of parents' emotions. Journal of Nonverbal Behavior, 33(2), 121-140.

Dunsmore, J. y Karn, M. (2001). Mothers' beliefs about feelings and children's emotional understanding. Early Education and Development, 12(1), 117-138.

Eisenberg, N., Cumberland, A. y Spinrad, T. (1998). Parental socialization of emotion. Psychological Inquiry, 9(4), 241-273.

Eisenberg, N., Spinrad, T. y Cumberland, A. (1998). The socialization of emotion: Replies to commentaries. Psychological Inquiry, 9(4), 317-333. 
Fernández, I. (2005). Las emociones como representaciones compartidas su anclaje sociocultural. En D. Páez., I. Fernández, S. Ubillos y E. Zubieta (Coords.), Psicología social, cultura y educación. (pp. 455-468). Madrid: Pearson.

Finney, S. y DiStefano, C. (2006). Non-normal and categorical data in structural equation modeling. En G. Hancock \& R. Mueller, Structural equation modeling: a second course (pp. 269-314). Greenwich, Connecticut: Information Age Publishing.

García-Coll, C. \& Pachter, L. (2002). Ethnic and minority parenting. En M. Bornstein (Ed.). Handbook of parenting: Vol. 4. Social conditions and applied parenting. (pp. 1-20). Mahwah, NJ: Erlbaum.

González, A. (Ed.). (2012). The emotions and cultural analysis. London: Ashgate.

Gottman, J., Katz, L. y Hooven, C. (1997). Meta-emotion: How families communicate emotionally. Mahwah, NJ: Lawrence Erlbaum Associates, Inc.

Gutentag, T., Halperin, E., Porat, R., Bigman, Y. \& Tamir, M. (2016). Successful emotion regulation requires both conviction and skill: beliefs about the controllability of emotions, reappraisal., and regulation success. Cognition and Emotion. 31(6), 1225-1233. doi: 10.1080/02699931.2016.1213704.

Halberstadt, A., Dunsmore, J., Bryant, A., Parker, A., Beale, K. \& Thompson, J. (2013). Development and validation of the Parents' Beliefs about Children's Emotions Questionnaire. Psychological Assessment, 25(4), 1195-1210.

Halberstadt, A., Dunsmore, J., McElwain, N., Eaton, K. y McCool, A. (2001). Parents' beliefs about negative emotions. Unpublished questionnaire, North Carolina State University.

Halberstadt, A., Dunsmore, J., Parker, A., Beale, K., Thompson, J., \& Bryant, A. (2008a). Parents' beliefs about children's emotions. Unpublished questionnaire.

Halberstadt, A., Thompson, J., Parker, A. \& Dunsmore, J. (2008b). Parents' emotion-related beliefs and behaviours in relation to children's coping with the 11 September 2001 terrorist attacks. Infant and Child Development, 17(6), 557-580.

Halberstadt, A. y Lozada, F. (2011). Emotion development in infancy through the lens of culture. Emotion Review, 3(2), 158-168.

Halberstadt, A., Riquelme, E. y Oertwig, D. (s.f.). Similarities and differences in parents' beliefs about emotion across Chilean cultures. Manuscrito presentado para su publicación.

Hakim-Larson, J., Parker, A., Lee, C., Goodwin, J. y Voelker, S. (2006). Measuring parental metaemotion: Psychometric properties of the emotion-related parenting styles self-test. Early Education and Development, 17(2), 229-251.

Hu, L. y Bentler, P. (1999). Cutoff criteria for fit indexes in covariance structure analysis: Conventional criteria versus new alternatives. Structural Equation Modeling, 6(1), 1-55.

Jaramillo, O. (2013). Las creencias sobre las emociones en familias antioqueñas. Revista de la Facultad de Trabajo Social, 29(29), 195-216.

Jöreskog, K. (1971). Simultaneous factor analysis in several populations. Psychometrika, 36(4), 409426.

Jöreskog, K. y Sörbom, D. (1993). LISREL 8: Structural equation modeling with the SIMPLIS command language. Hillsdale, NJ: Lawrence Erlbaum Associates Publishers.

Keel, S. (2016). Socialization: Parent-child interaction in everyday life. New York: Routledge.

Lagacé-Séguin, D. y Coplan, R. (2005), Maternal emotional styles and child social adjustment: Assessment, correlates, outcomes and goodness of fit in early childhood. Social Development, 14(4), 613-636. doi:10.1111/j.1467-9507.2005.00320.x.

Lozada, F. Halberstadt, A., Craig, A., Dunsmore, J. y Dennis, P. (2016). Parents' beliefs about children's emotions and their conversations with children. Journal of Child and Family Studies, 25(5), 1525-1538. doi: 10.1007/s10826-015-0325-1.

Matsumoto, D. (1993). Ethnic differences in affect intensity, emotion judgments, display rule attitudes, and self-reported emotional expression in an American sample. Motivation and 
Estudios Pedagógicos XLV, Nº 2: 61-81, 2019

CREENCIAS DE LOS ADULTOS SOBRE LA EMOCIÓN DE LOS NIÑOS: UNA PROPUESTA DE EXPLORACIÓN EN CONTEXTOS DE DIVERSIDAD CULTURAL

Emotion, 17(2), 107-123.

McDonald, R. (1999). Test theory: A unified treatment. Mahwah, N.J.: L. Erlbaum Associates.

McGillicuddy-DeLisi, A. (2009). Parents' beliefs and children's personal-social development. En I. Sigel, A. McGillicuddy-DeLisi y J. Goodnow (Eds.), Parental belief systems (pp. 115-142). New York: Psychology Press.

Mesquita, B., Marinetti, C. y Delvaux, E. (2012). The social psychology of emotions. En S. Fiske y C. McCrae (Eds.), Handbook of social cognition (pp. 297-317). New York: Sage.

Muroff, J., Spencer, M., Ross, A., Williams, D., Neighbors, H. y Jackson, J. (2014). Race, gender, and conceptualizations of fear. Professional Psychology, Research and Practice, 45(3), 153-162.

Muthén, L. y Muthén, B. (2007). Mplus User's Guide (Sixth edition). Los Angeles, CA: Muthén \& Muthén.

Nelson, J., Leerkes, E., O'Brien, M., Calkins, S. \& Marcovitch, S. (2012). African american and european american mothers' beliefs about negative emotions and emotion socialization practices. Parenting, science and practice, 12(1), 22-41. doi:10.1080/15295192.2012.638871.

Nicolaisen, I. (1995). Persons And nonpersons: Disability and personhood among the Punan Bah of Central Borneo. En B. Ingstad y S. Reynolds (Eds.), Disability and Culture (pp. 38-55). Los Angeles: University of California Press.

Ogbu, J. \& Simons, H. (1998). Voluntary and involuntary minorities: A cultural-ecological theory of school performance with some implications for education. Anthropology y Education Quarterly, 29(2), 155-188. doi: https://doi.org/10.1525/aeq.1998.29.2.155.

Parker, A., Halberstadt, A., Dunsmore, J., Townley, G., Bryant, A., Thompson, J. y Beale, K. (2012). Emotions are a window into one's heart: A qualitative analysis of parental beliefs about children's emotions across three ethnic groups. Monographs of the Society for Research in Child Development, 77(3), 1-144.

Quilaqueo, D., Quintriqueo, S., y Torres, H. (2016). Características epistémicas de los métodos educativos mapuches. Revista Electrónica de Investigación Educativa, 18(1), 153-165.

Ramsden, S. y Hubbard, J. (2002). Family expressiveness and parental emotion coaching: Their role in children's emotion regulation and aggression. Journal of abnormal child psychology, 30(6), 657-667.

Raval V. y Martini T. (2009). Maternal socialization of children's anger, sadness, and physical pain in two communities in Gujarat, India. International journal of behavioral development, 33(3), $215-229$

Raval, V., Raval, P., Salvina, J., Wilson, S. y Writer, S. (2012). Mothers' socialization of children's emotion in India and the USA: A cross- and within-culture comparison. Social Development, 22(3), 467-484.

Riquelme, E. y Montero, I. (2013) Improving emotional competence through mediated reading: Short term effects of a children's literature program. Mind, Culture, and Activity, 20(3), 226-239.

Riquelme, E. y Munita, F. (2017). The mediated reading of children's literature as "paradigmatic scenario" in the development of emotional competence: Explore the fictional line to change the real script. Psicología, Teoría \& Pesquisa. Manuscrito presentado para su publicación.

Riquelme, E., Munita, F., Jara, E. y Montero, I. (2013). Reconocimiento facial de emociones y desarrollo de la empatía mediante la lectura mediada de literatura infantil. Cultura y Educación, 25(3) 375-388.

Riquelme, E., Quilaqueo, D., Quintriqueo, S. y Loncón, E. (2016). Predominancia de la educación emocional occidental en contexto indígena: necesidad de una educación culturalmente pertinente. Psicologia Escolar e Educacional, 20(3), 523-532.

Riquelme, E., Lavoie, G., Quilaqueo, D. y Quintriqueo, S. (2017). Emotion and exclusion: key ideas from Vygotsky to review our role in a school with a cultural diversity setting. Revista Brasileira de Educação Especial. Manuscrito presentado para su publicación.

Rimes, K. y Chalder, T. (2010). The beliefs about emotions scale: Validity, reliability and sensitivity 
to change, Journal of psychosomatic research, 68(3), 285-292.

Rogers, M., Halberstadt, A., Castro, V., MacCormack, J. y Garrett-Peters, P. (2016). Maternal emotion-related beliefs, socialization behaviors, and regulatory skills in relation to children's emotion regulation and lability. Emotion, 16(2), 280-291. doi: 10.1037/emo0000142.

Rosso, L. (2012). Parents' beliefs about emotion and children's subsequent coping strategies as influences on children's level of peer victimization. Psychological Science Undergraduate Honors Theses. Paper 2.

Sala, J. y Abarca, M. (2001). La educación emocional en el currículum. Teoría de la Educación, 13, 209-232.

Satorra, A. y Bentler, P. (2001). A scaled difference chi-square test statistic for moment structure analysis. Psychometrika, 66(4), 507-514.

Shipman, K. y Zeman, J. (2001). Socialization of children's emotion regulation in mother-child dyads: A developmental psychopathology perspective. Development and Psychopathology, 13(2), 317-336.

Steiger, J. (2007). Understanding the limitations of global fit assessment in structural equation modeling. Personality and Individual Differences, 42(5), 893-898.

Stelter, R. y Halberstadt, A. (2011). The interplay between parental beliefs about children's emotions and parental stress impacts children's attachment security. Infant and Child Development, 20(3), 272-287. doi:10.1002/icd.693.

Sigel, I. y Kim, M. (1996). The answer depends on the question. En S. Harkness \& C. Super (Eds.), Parents' cultural belief systems: Their origins, expressions, and consequences (pp. 83-120). New York: The Guilford Press.

Tamir, M., Srivastava, S., John, O. \& Gross, J. (2007). Implicit theories of emotion: Affective and social outcomes across a major life transition. Journal of personality and social psychology, 92(4), 731-744.

Tamir, M., Schwartz, S., Cieciuch, J., Riediger, M., Torres, C., Scollon, C., Dzokoto, V., Zhou, X. y Vishkin, A. (2016). Desired emotions across cultures: A value-based account. Journal of Personality and Social Psychology, 111(1), 67-82.

Tsai, J. (2007). Ideal affect: Cultural causes and behavioral consequences. Perspectives on Psychological Science, 2(3), 242-259.

Tsai, J., Louie, J., Chen, E. y Uchida, Y. (2007). Learning what feelings to desire: Socialization of ideal affect through children's storybooks. Personality and Social Psychology Bulletin, 33(1), 17-30. doi:10.1177/0146167206292749.

Uzbilir, G. (2016). The relationship between mothers' and fathers' beliefs about children's emotions and emotion socialization practices. Tesis doctoral sin publicar. Estambul: İstanbul Bilgi Üniversitesi.

Veilleux, J., Salomaa, A., Shaver, J., Zielinski, M., y Pollert, G. (2015). Multidimensional assessment of beliefs about emotion: Development and validation of the emotion and regulation beliefs scale. Assessment, 22(1), 86-100.

Wege, B., Sánchez, M., Friedlmeier, W., Mihalca, L., Goodrich, E. y Corapci, F. (2014). Emotion displays in media: a comparison between American, Romanian, and Turkish children's storybooks. Frontiers in Psychology, 5, 600.

Wong, M., Diener, M. y Isabella, R. (2008). Parents' emotion related beliefs and behaviors and child grade: Associations with children's perceptions of peer competence. Journal of Applied Developmental Psychology, 29(3), 175-186. doi: 10.1016/j.appdev.2008.02.003.

Wong, M., McElwain, N. y Halberstadt, A. (2009). Parent, family, and child characteristics: Associations with mother- and father-reported emotion socialization practices. Journal of Family Psychology, 23(4), 452-463. 
